\title{
ON REAL IRREDUCIBLE REPRESENTATIONS OF LIE ALGEBRAS
}

\author{
NAGAYOSHI IWAHORI
}

\section{$\S 1$. Introduction}

Let us consider the following two problems:

Problem A. Let $\mathrm{g}$ be a given Lie algebra over the real number field $R$. Then find all real, irreducible representations of $\mathrm{g}$.

Problem B. Let $n$ be a given positive integer. Then find all irreducible subalgebras of the Lie algebra $\mathrm{gl}(n, R)$ of all real matrices of degree $n$.

In a beautiful and fundamental paper [1], E. Cartan solved completely the Problem $\mathrm{B}$, in the sense that he gave a method to determine all the subalgebras of $g r(n, R)$ by a finite process, and determined them actually for the case $n \leqq 12$ for which he gave a table. As we shall see in $\S 6,7$, the Problem $\mathrm{A}$ is reduced to the one to find all complex irreducible representations and to distinguish among them those representations which are of the first class, and then the Problem $\mathrm{A}$ is easily reduced to the reductive case, i.e. to the case where $\mathfrak{B}$ is reductive. As a reductive Lie algebra is a direct sum of simple Lie algebras, the Problem $A$ can be further reduced to the case where $\mathfrak{g}$ is simple, as we shall see later. Now if the Problem A could be solved for every Lie algebra 8 , then one has only to look at the table to solve $B$. In analysing [1] closely, we notice that E. Cartan solved the Problem B by this principle. In several places of [1], E. Cartan has recourse to verifications for each type of simple Lie algebras A, B, C, D and the results of verifications for exceptional cases are stated without proof.

In the present paper, we shall solve the Problem A by the above mentioned principle and reestablish the results of [1]. The knowledge of [1] is not presupposed for the reader. Where $\mathrm{E}$. Cartan had recourse to verifications for each type of simple algebras, we shall be able to obtain the corresponding results by general considerations.

Received May 31, 1958. 


\section{§. Complex conjugates of complex vector spaces}

For later use, we state here some facts about "complex conjugates" of complex vector spases. Let $V, U$ be finite dimensional vector spaces over the complex number field $C$. A mapping $f: V \rightarrow U$ is called anti-linear if

$$
f(\alpha x+\beta y)=\bar{\alpha} f(x)+\bar{\beta} f(y)
$$

for every $\alpha, \beta \in C$ and $x, y \in V$. In particular an anti-linear mapping from $V$ into $C$ is called an anti-linear form on $V$. Let us denote by $V^{(*)}$ the set of all anti-linear forms on $V$. Then by the operations

$$
\left(f_{1}+f_{2}\right)(x)=f_{1}(x)+f_{2}(x),(\alpha f)(x)=\alpha \cdot f(x)
$$

for $f_{1}, f_{2}, f \in V^{(*)}, x \in V, \alpha \in C, V^{(*)}$ becomes a complex vector space and dis $V^{(*)}=\operatorname{dim} V$.

Now let us denote by $\bar{V}$ the dual vector space of the complex vector space $V^{(*)}$, i.e. the vector space consisting of all linear forms on $V^{(*)}$. Then every $x \in V$ determines an element $\bar{x} \in \bar{V}$ as follows :

$$
(\bar{x}, f)=(x, f)=f(x) \quad \text { for every } f \in V^{(*)}
$$

and the mapping $x \rightarrow \bar{x}$ is a one-to-one, anti-linear mapping from $V$ onto $\bar{V}$. Moreover, if $A$ is a linear endomorphism of the vector space $V$, then $A$ determines a linear endomorphism $\bar{A}$ of $\bar{V}$ as follows : $\bar{A} \bar{x}=\overline{A x}$ for every $x$ in $V$. Then the mapping $A \rightarrow \bar{A}$ is a one-to-one anti-linear mapping from the vector space $\mathfrak{g}(V)$ of all linear endomorphisms of the complex vector space $V$ onto: $\operatorname{gr}(\bar{V})$.

We note that if $\left(e_{i}\right)$ is a base of $V$, then $\left(\bar{e}_{i}\right)$ is a base of $\bar{V}$ and the matrix of $A \in \mathfrak{g l}(V)$ with respect to $\left(e_{i}\right)$ is the complex conjugate of the matrix of $\bar{A} \in g l(\bar{V})$ with respect to $\left(\bar{e}_{i}\right)$. We shall call $\bar{V}, \bar{x}, \bar{A}$ the complex conjugates of $V, x, A$ respectively.

\section{§3. Scalar restrictions and scalar extentions}

Let $V$ be a vector space over $C$. Then $V$ can be regarded in a natural way also a vector space over $R$. We denote this real vector space by $V_{R}$ and call it the scalar restriction of $V$ to the real number field $R$. Note that $V$ and $V_{R}$ coincide as a set. Now if $A$ is a linear endomorphism of the complex vector space $V$, then $A$ induces naturally a linear endomorphism $A_{R}$ of the real 
vector space $V_{R}$.

If $(\rho, V)$ is a complex representation of a real Lie algebra $g$, then $\left(\rho_{R}, V_{R}\right)$ is a real representation of $g$, where $\rho_{R}(X)=(\rho(X))_{R}$ for every $X \in g$. We shall call the real representation $\left(\rho_{R}, V_{R}\right)$ the scalar restriction of the complex representation $(\rho, V)$.

Now let $E$ be a vector space over $R$. Then we denote by $E^{c}$ the complex vector space which is obtained from $E$ by extending the ground field $R$ to $C$. If $A$ is a linear endomorphism of $E$, then $A$ is extended uniquely to a linear endomorphism $A^{C}$ of $E^{C}$.

If $(d, E)$ is a real representation of a real Lie algebra $g$, then $\left(d^{(i)}, E^{(i)}\right)$ is a complex representation of $\mathfrak{g}$, where $d^{\prime}(X)=(d(X))^{c}$ for every $X \in \mathfrak{g}$. We shall call the complex representation $\left(d^{c}, E^{r}\right)$ the scalar extension of the real representation $(d, E)$.

\section{$\S 4$. Conjugate representations}

Let $(\rho, V)$ be a complex representation of a real Lie algebra 9 . Then we can form another complex representation $(\bar{\rho}, \bar{V})$ of $\mathfrak{g}$, where $\bar{V}$ is the complex conjugate of the complex vector space $V$, and $\bar{\rho}$ is defined by $\bar{\rho}(X)=\rho(X)$ for every $X \in \mathrm{g}$. Since $g$ is real Lie algebra, $(\bar{\rho}, \bar{V})$ becomes a complex representation of $g$. We note that the scalar restrictions $\rho_{R}, \bar{\rho}_{R}$ are equivalent real representation of $g$. In fact the mapping $x \rightarrow \bar{x}$ from $V$ onto $\bar{V}$ gives the equivalence of $V_{R}$ and $\bar{V}_{R}$. Now let $(\rho, V),(\sigma, U)$ be two complex representations of $g$. If $(\bar{\rho}, \bar{V})$ is equivalent to $(\sigma, U)$, then we shall say that $(\rho, V)$ is conjugate to $(\sigma, U)$ and denote it by $\vec{\rho} \sim \sigma$. In particular, if $\vec{\rho} \sim \rho$, then we say $\rho$ self-conjugate. If $\bar{\rho} \sim \sigma$, then we have easily $\rho \sim \bar{\sigma}$, so the relation of "conjugate" is symmetric. Let us note that a complex representation $(\rho, V)$ is conjugate to $(\sigma, U)$ if and only if there exists a one-to-one anti-linear mapping $f$ from $V$ onto $U$ such that

$$
f \circ \rho(X)=\sigma(X) \circ f
$$

for every $X \in \Omega$. In fact, if $\bar{\rho} \sim \sigma$, then there is a linear isomorphism $\varphi: \vec{V} \rightarrow U$ such that $\varphi \circ \bar{\rho}(X)=\sigma(X) \circ \varphi$ for every $X \in g$. Define the mapping $f$ by $f(x)$ $=\varphi(\bar{x})$, then $f$ has all the desired properties. The converse is shown analogously. 
In particular a complex representation $(\rho, V)$ is self-conjugate if and only if there is a one-to-one anti-linear mapping $J$ from $V$ onto itself (we shall call such a mapping $J$ anti-linear automorphism of $V$ ) such that

$$
J \circ \rho(X)=\rho(X) \circ J
$$

for every $X \in \mathfrak{g}$, i.e. $J$ is commutative with every $\rho(X)(X \in \mathfrak{g})$. In this case we say also that $J$ is invariant by $\rho$ or that $\rho$ leaves $J$ invariant.

Now let us remark that our notion of conjugate or self-conjugate representation coincides with the notion of "correlatif" or "auto-correlatif" of E. Cartan [1] respectively, if $g$ is a semi-simple Lie algebra over $R$. To this purpose we shall prove the following

Lemma 1. Let $\mathfrak{g}$ be a semi-simple Lie algebra over $R$ and $(\rho, V),(\sigma, U)$ be two complex representations of $\mathrm{g}$. Then $(\rho, V)$ is equivalent to $(\sigma, U)$ if and only if the characteristic polynomials of both representations coincide, i.e.

$$
\operatorname{det}(t I-\rho(X))=\operatorname{det}(t I-\sigma(X))
$$

for every $X \in \mathrm{Q}$, where $t$ is an indeterminate and $I$ is the identity operator on $V$ or $U$.

Proof. Assume that (1) hold for every $X \in g$ and let us prove that $\rho \sim \sigma$. Let $\mathfrak{g}^{c}$ be the complex form of $g$ and $\mathfrak{h}$ be a Cartan subalgebra of $\mathfrak{g}$. Then $\mathfrak{h}^{\prime}$ is a Cartan subalgebra of $g^{c}$. Now every complex representation $(\rho, V)$ of $\mathfrak{g}$ can be extended uniquely to the complex representation of $g^{c}$ which we also denote by $(\rho, V)$. Then as is easily seen, (1) holds for every $X \in g^{\prime}$. Now let $\Lambda_{1}, \ldots, A_{r}$ and $\Lambda_{1}^{\prime}, \ldots, \Lambda_{s}^{\prime}$ be the system of weights of representation $(\rho, V),(\sigma, U)$ respectively with respect to the Cartan subalgebra $\mathfrak{h}^{c}$. Then by (1) we have

$$
\prod_{i=1}^{r}\left(t-\Lambda_{i}(H)\right)^{m_{i}}=\prod_{j=1}^{s}\left(t-\Lambda_{j}^{\prime}(H)\right)^{n_{j}}
$$

for every $H \in \mathfrak{h}^{c}$, where $m_{i}, n_{j}$ are the multiplicities of $\Lambda_{i}, \Lambda_{j}^{\prime}$ respectively. Then we have $r=s$ and $A_{1}, \ldots, A_{r}$ coincide with $A_{1}^{\prime}, \ldots, A_{r}^{\prime}$ together with their multiplicities up to their order. Then, the highest weights of every irreducible component of $(\rho, V)$ and $(\sigma, U)$ must coincide together with their multiplicities. Thus we have $\rho \sim \sigma$ as representations of $\beta^{c}$. Then we have $\rho \sim \sigma$ as representations of $g$. 
The converse assertion is trivial. So we have completed the proof.

Corollary. Let $g$ be a semi-simple Lie algebra over $R$, and $(\rho, V),(\sigma, U)$ be two complex representation of $\mathrm{g}$. Then $\bar{\rho} \sim \sigma$ if and only if the coefficients of the characteristic polynomials of $\rho(X), \sigma(X)$ are complex conjugate of each other for every $X \in g$. In particular, $\rho$ is self-conjugate if and only if the coefficients of the characteristic polynomial of $\rho(X)$ are all real numbers.

In [1], E. Cartan has defined the notion of "correlatif" or "auto-correlatif" using the characteristic polynomials of representations. The relation of this notion to our notion of conjugateness or self-conjugateness is shown in the above corollary.

\section{$\S 5$. Fundamental theorem of E. Cartan}

We are now in an appropriate position to explain the fundamental theorem of E. Cartan connecting real, irreducible representations with complex, irreducible representations. Now let $g$ be a Lie algebra over $R$. Let us denote by $R_{n}(g)$ the set of all real, irreducible representation classes of $g$ of degree $n$, and by $C_{n}(g)$ the set of all complex, irreducible representation classes of $g$ of degree $n$. We also denote by $R_{n}^{L}(\mathrm{~g}), R_{n}^{I L}(\mathrm{~g})$, the following subsets of $R_{n}(\mathrm{~g})$ :

$$
\begin{aligned}
& R_{n}^{l}(\mathfrak{g})=\left\{[d] \in R_{n}(\mathfrak{g}) ; d^{C} \text { is irreducible }\right\} \\
& R_{n}^{I l}(\mathfrak{g})=\left\{[d] \in R_{n}(\mathfrak{g}) ; d^{C} \text { is reducible }\right\}
\end{aligned}
$$

where $[d]$ means the representation class containing $d$. If $[d] \in R_{n}^{l}(g)$ or $\in R_{n}^{I l}(g)$, then $[d]$ and $d$ are called of first class or second class respectively. We also denote by $C_{n}^{I}(\mathrm{~g}), C_{n}^{I I}(\mathrm{~g})$ the following subsets of $C_{n}(\mathfrak{g})$ :

$$
\begin{aligned}
& C_{n}^{I}(g)=\left\{[\rho] \in C_{n}(g) ; \rho_{R} \text { is reducible }\right\} \\
& C_{n}^{I I}(g)=\left\{[\rho] \in C_{n}(g) ; \rho_{R} \text { is irreducible }\right\}
\end{aligned}
$$

If $[\rho] \in C_{n}^{I}(g)$ or $\in C_{n}^{I I}(g)$, then $[\rho]$ and $\rho$ are called of first class or second class respectively. Then we have obviously

$$
\begin{aligned}
& R_{n}(\mathfrak{g})=R_{n}^{I}(\mathrm{~g}) \cup R_{n}^{I l}(\mathrm{~g}), R_{n}^{l}(\mathrm{~g}) \cap R_{n}^{I I}(\mathfrak{g})=\text { empty set, } \\
& C_{n}(\mathfrak{g})=C_{n}^{I}(\mathrm{~g}) \cup C_{n}^{I I}(\mathfrak{g}), C_{n}^{I I}(\mathfrak{g}) \cap C_{n}^{I I}(\mathfrak{g})=\text { empty set. }
\end{aligned}
$$

Now let us associate to an irreducible real representation $(d, E)$ of first class, an irreducible complex representation $\left(d^{\prime}, E^{\prime}\right)$. Since from $d_{1} \sim d_{2}$, we have 
$d_{1}^{c} \sim d_{2}^{c}$, we have a mapping

$$
\Psi_{1}:[d] \rightarrow\left[d^{c}\right]
$$

from $R_{n}^{I}(g)$ into $C_{n}(g)$.

If $(d, E)$ is an irreducible real representation of second class, then $\left(d^{c}, E^{c}\right)$ is reducible. Let $V$ be any invariant subspace of $E^{c}$ such that $V \neq E^{c}, V \neq(0)$. Then, denoting by $x \rightarrow \bar{x}$ the anti-linear automorphism of $E^{c}$ determined by $E$ (i.e. if $x=y+\sqrt{-1} z, y \in E, z \in E$, then $\bar{x}=y-\sqrt{-1} z$ ) and by $\bar{V}$ the image of $V$ under this mapping $x \rightarrow \bar{x}$, we have

$$
E^{c}=V+\bar{V}, V \cap \bar{V}=(0) .
$$

In fact, since $\overline{V+\bar{V}}=V+\bar{V}$, we have $V+\bar{V}=F+\sqrt{-1} F$ where $F=(V+\vec{V})$ $\cap E$. $^{1)} \quad$ Then $F \neq(0)$ is an invariant subspace of $E$. Hence we have $F=E$ and $V+\bar{V}=E^{C}$. Similarly we have $V \cap \bar{V}=(0)$. Thus (2) is proved. Now $V$ is irreducible. In fact, if $V$ contains an invariant complex subspace $U$ such that $U \neq V, U \neq(0)$, then $U+\bar{U} \neq E^{c}$ which contradicts to (2). Similarly $\bar{V}$ is irreducible. The irreducible representations induced by $d^{c}$ on $V, \bar{V}$ are, as is seen easily, conjugate to each other. Thus, we have $\operatorname{dim}_{R} E=2 \operatorname{dim}_{c} V$, i.e. if $[d] \in R_{n}^{I l}(\mathrm{~g})$, then $n$ must be an even integer.

Let us associate to $[d] \in R_{n}^{l l}(g)$ the irreducible complex representation class $[\rho] \in C_{n / 2}(\mathrm{~g})$, where $\rho$ is the representation induced by $d^{c}$ on $V$ or on $\bar{V}$ as above. $[\rho]$ is determined up to conjugate representation class. Let us introduce an equivalence relation $\approx$ in the set $C_{n / 2}(g)$ by

$$
\left[\rho_{1}\right] \approx\left[\rho_{2}\right] \text { if }\left[\rho_{1}\right]=\left[\rho_{2}\right] \text { or }\left[\bar{\rho}_{1}\right]=\left[\rho_{2}\right]
$$

and denote by $\hat{C}_{n / 2}(g)$ the set of all equivalence class in $C_{n / 2}(g)$ with respect to the equivalence relation $\approx$. Then by the above mapping

$$
R_{n}^{I l}(\mathfrak{g}) \ni[d] \rightarrow[\rho] \in C_{n / 2}(\mathfrak{g})
$$

there is introduced a mapping

$$
\Psi_{2}:[d] \rightarrow(\approx) \text {-equivalence class of }[\rho]
$$

from $R_{n}^{I I}(\mathfrak{g})$ into $\hat{C}_{n / 2}(\mathfrak{g})$.

1) In general, a complex subspace $W$ of $E^{r}$ has a form $W=F+\sqrt{-1} F$ (where $F$ is a real subspace of $E$ ), if and only if $W=\bar{W}$. Moreover, if $W=\bar{W}$, then $F$ is given by $F$ $=W \cap E$. 
Now let us explain other' mappings $\Psi_{3}, \Psi_{4}$. Let $(\rho, V)$ be an irreducible complex representation of first class. Then $\left(\rho_{R}, V_{R}\right)$ is reducible. Let $E$ be an invariant (real) subspace of $V_{R}$ such that $E \neq V_{R}, E \neq(0)$. Then $E+V-1 E$ and $E \cap \sqrt{-1} E$ are invariant (complex) subspaces of $V$ and we have $E+$ $\sqrt{-1} E \neq(0), E \cap \sqrt{-1} E \neq V$. Hence we have

$$
V_{R}=E+\sqrt{ }-1 E, E \cap \sqrt{ }-1 E=0 .
$$

Now $E$ is irreducible. In fact if $E$ contains an invariant (real) subspace $F$ such that $F \neq E, F \neq(0)$, then we have $V \neq F+\sqrt{ }-1 F$ which contradicts to (3). Similarly $\sqrt{-1} E$ is irreducible. Moreover the irreducible real representations induced by $\rho_{R}$ on $E$ and $\sqrt{ }-1 E$ are equivalent to each other. In fact, the one-to-one linear mapping $x \rightarrow \sqrt{ }-1 \quad x$ from $E$ onto $\sqrt{ }-1 E$ gives the equivalence of $E$ and $\sqrt{ }-1 E$. Let $d$ be the irreducible real representation induced by $\rho_{R}$ on $E$ or on $\sqrt{-1} E$, then we have a mapping

$$
F_{3}:[\rho] \rightarrow[d]
$$

from $C_{n}^{\prime}(\mathfrak{g})$ into $R_{n}(\mathfrak{g})$.

Now let $(\rho, V)$ be an irreducible complex representation of second class. Then $\left(\rho_{R}, V_{R}\right)$ is an irreducible real representation of degree $2 n$. Moreover, as is remarked in $\S 4, \rho$ and $\bar{\rho}$ give equivalent real representations $\rho_{R}, \bar{\rho}_{R}$. Hence there is induced a mapping

$$
\Psi_{4}:(\approx) \text {-equivalence class of }[\rho] \rightarrow\left[\rho_{R}\right]
$$

from $\hat{C}_{n}(g)$ into $R_{2 n}(\mathrm{~g})$. We denote by $\hat{C}_{n}^{\prime \prime}(\mathrm{g})$ the subset of $\hat{C}_{n}(\mathrm{~g})$ consisting of $(\approx)$-equivalenct classes containing an irreducible complex representation of second class.

Now under these preparations, we can state the fundamental theorem of E. Cartan as follows:

THEOREM 1. (i) $\Psi_{1}$ is a one-to-one mapping from $R_{n}^{\prime}(g)$ onto $C_{n}^{\prime}(g)$. $\Psi_{3}$ is a one-to-one mapping from $C_{n}^{l}(g)$ onto $R_{n}^{\prime}(g) . \quad \Psi_{1}$ and $\Psi_{3}$ are the inverse mappings of each other. (ii) $\Psi_{2}$ is a one-to-one mapping from $R_{2 n}^{I I}(g)$ onto $\hat{C}_{n l}^{l l}(\mathrm{~g}) . \Psi_{4}$ is a one-to-one mapping from $\hat{C}_{n}^{\prime \prime}(\mathrm{g})$ onto $R_{2 n}^{\prime \prime}(\mathrm{g})$. $\Psi_{2}$ and $\Psi_{1}$ are the inverse mappings of each other.

Proof. (i) Let $[d] \in R_{n}^{\prime}(g)$. Then $\Psi_{1}([d])=\left[d^{\prime}\right]$. Let $E$ be the representation space of the representation $d$. Then $E^{c}$ is the representation space of 
$d^{\prime}$. Then, putting $d^{\prime}=\rho, E^{\prime}=V$, let us show that $\rho_{R}$ is reducible. In fact, we have $V_{R}=E+\sqrt{-1} E, E \cap \sqrt{-1} E=(0)$, and $E$ is an invariant subspace of $V_{R}$. Thus $d^{\prime}$ belongs to $C_{n}^{\prime}(\mathfrak{g})$ and $\Psi_{1}\left(R_{n}^{l}(\mathfrak{g})\right) \subset C_{n}^{\prime}(\mathfrak{g})$. Moreover, since $\rho_{R}$ induces an irreducible real representation $d$ on $E$, we have

$$
\Psi_{3} \Psi_{1}([d])=[d]
$$

for every $[d] \in R_{n}^{I}(g)$.

Next let $[\rho] \in C_{n}^{I}(\mathfrak{g})$. Let $V$ be the representation space of $\rho$. Since $V_{R}$ is reducible, there is an invariant subspace $E$ of $V_{R}$ such that $E \neq V_{R}, E \neq(0)$. Then we have $V_{R}=E+\sqrt{ }-1 E, E \cap \sqrt{-1} E=(0)$ by (3). Then $V$ can be regarded as $E^{r}$. Denoting by $d$ the irreducible real representation induced by $\rho_{R}$ on $E$, we have then $d^{c}=\rho$. Then we have $[d] \in R_{n}^{I}(g)$. Thus we have shown that $\Psi_{3}\left(C_{n}^{I}(g)\right) \subset R_{n}^{I}(g)$ and

$$
\Psi_{1} \Psi_{3}([\rho])=[\rho]
$$

for every $[\rho] \in C_{n}^{l}(\mathrm{~g})$. Thus (i) is proved. (ii) Let $[d] \in R_{2 n}^{I I}(\mathrm{~g})$. Let $E$ be the representation space of the representation $d$. Then $E^{C}=V$ contains an irreducible, invariant subspace $U$ such that $V=U+\bar{U}, U \cap \bar{U}=(0)(\bar{U}$ is the complex conjugate of $U$ with respect to the complex conjugation of $E^{r}$ with respect to $E$ ). Let $\rho$ be the irreducible representation induced by $d^{c}$ on $U$. Let us show that $\left(\rho_{R}, U_{R}\right)$ is an irreducible real representation. In fact, if $U_{R}$ contains an invariant subspace $F$ such that $F \neq U_{R}, F \neq(0)$, we have $F+\bar{F}=F_{0}$ $+\sqrt{-1} F_{0}$ where $F_{0}=(F+\bar{F}) \cap E$. Then $F_{0}$ is an invariant subspace of $E$ such that $F_{0} \neq E, F_{0} \neq(0)$. This contradicts to the fact that $E$ is irreducible. Thus we have $[\rho] \in C_{n}^{\prime \prime}(g)$. Let us show moreover that $\rho_{R} \sim d$. In fact, let us associate to a vector $u \in U$ a vector $\varphi(u)=u+\bar{u} \in E^{C}$. Then $\varphi(u) \in E$. The mapping $\varphi: U \rightarrow E$ thus defined induces a linear mapping $\varphi: U_{R} \rightarrow E$. Since every element $x \in E$ is expressible uniquely as $x=u+\bar{u}(u \in U), \varphi$ is a linear isomorphism from $U_{R}$ onto $E$. Now let $X$ be any element of the Lie algebra $g$. Then we have

$$
\varphi \circ \rho_{R}(X)=d(X) \circ \varphi
$$

since $U$ and $\bar{U}$ are invariant subspaces and $d(X)$ commute with the mapping $x \rightarrow \bar{x}$. Thus we have $\rho_{R} \sim d$, and we have proved that $\Psi_{2}\left(R_{2 n}^{\prime \prime}(g)\right) \subset \hat{C}_{n}^{\prime \prime}(\beta)$ and

$$
\Psi_{1} \Psi_{2}([d])=[d]
$$


for every $[d] \in R_{2 n}^{\prime \prime}(\Omega)$.

Next, let $[\rho] \in C_{n}^{\prime \prime}(3)$. Let $V$ be the representation space of the representation $\rho$. Put $E=V_{R}$ and $d=\rho_{R}$, then $(d, E)$ is an irreducible real representation of 9 . Let us denote the linear automorphism $x \rightarrow \sqrt{-1} x$ of the real vector space $E$ by $\mathscr{D}$. Then $\mathscr{D}^{2}=-I$ ( $I$ means the identity operator of $E$ ). Let $U_{+}$, $U_{-}$be the eigen space of the linear automorphism $\Phi^{\prime}$ of the complex vector space $E^{\prime}$ associated to eigen values $\sqrt{ }-1,-\sqrt{ }-1$ respectively:

$$
U_{-}=\left\{x \in E^{c} ; \Phi^{\prime}(x)=\sqrt{ }-1 \quad x\right\}, U_{-.}=\left\{x \in E^{\prime} ; \Phi^{\prime}(x)=-\sqrt{ }-1 x\right\} .
$$

Then we have $E^{\prime}=U_{+}+U_{-}, U_{+} \cap U_{-}=(0)$. Let us denote by $x \rightarrow \bar{x}$ the com. plex conjugation of $E^{r}$ with respect to $E$. Then since $\mathscr{D}^{\prime}(\bar{x})=\mathscr{\Phi}^{c}(\bar{x})$ we have

$$
\bar{U}_{+}=U_{-} \text {. }
$$

Moreover $U_{+}, U_{-}$are invariant subspaces because $\emptyset$ commutes with every $d(X)$ $=\rho_{R}(X), X \in \mathfrak{g}$. Thus we have $[d] \in R_{2 n}^{I I}(\mathrm{~g})$, and then $U_{+}$and $U_{-}$are irreducible invariant subspaces of $E^{\Upsilon}$. Let us denote by $\rho_{1}$ the irreducible representation induced by $d^{c i}$ on $U_{+}$. Then $\rho \sim \rho_{1}$. In fact, an element $u=x+\sqrt{-1} y \in E^{r}$ $(x, y \in E)$ is in $U_{+}$if and only if $x=\mathscr{D}(y)$. Let us associate to an element $y$ of $V$ (we note that as a set $V=V_{R}=E$ ) the element $\Phi(y)+\sqrt{ }-1 y$ of $U_{+}$. Then we have a mapping $\varphi: y \rightarrow \emptyset(y)+\sqrt{-1} y$ from $V$ onto $U_{+}$. Obviously $\varphi$ is linear over $R$. Moreover $\varphi$ is linear over $C$, becuase we have $\varphi\left(\begin{array}{lll}\checkmark & y\end{array}\right)$ $=\varphi(\Phi y)=\mathscr{D}^{2} y+\sqrt{-1} \Phi(y)=-y+\sqrt{-1} \Phi(y)=\sqrt{-1} \varphi(y)$.

Moreover, $\varphi$ is an isomorphism. In fact, if $\varphi(y)=0$ we have $\emptyset(y)=0$, $y=0$, Thus $\varphi$ is a complex linear isomorphism from $V$ onto $U_{+}$. Now let $X$ be any element of $g$. Then, since $d=\rho_{R}$, we have $\varphi \circ \rho(X)=\rho_{1}(X) \circ \varphi$. Thus we have shown that $\Psi_{4}\left(\hat{C}_{n}^{I I}(g)\right) \subset R_{2 n}^{I I}(g)$ and that

$$
\Psi_{2} \Psi_{4}((\approx) \text {-equivalence class of }[\rho])=(\approx) \text {-equivalence class of }[\rho]
$$

for every $[\rho] \in C_{n}^{I I}(\S)$. Thus (ii) is proved.

Remark. Theorem 1 is also valid for associative algebras and Jordan algebras etc. over $R$.

\section{\$6. Reduction of the Problem (A) to the complex irreducible representations}

By theorem 1 we have to consider only complex irreducible representations 
exclusively. In the following we treat only complex representation, so we say simply representation instead of complex representation.

Now Problem $A$ is thus reduced to the following problems:

Problem $\left(A_{1}\right)$ : Find all irreducible (complex) representations of a given real Lie algebra 9.

Problem $\left(A_{2}\right)$ : Let $(\rho, V)$ be an irreducible (complex) representation of $\mathrm{g}$. Decide whether $\rho$ is of first class or of second class.

Now among these problems, Problem $\left(A_{1}\right)$ is equivalent to find all irreducible representations of the complex form $g^{\prime}$ of $g$. It is well-known that the problem of finding all irreducible representation of a given complex Lie algebra is reduced to the case of simple Lie algebras (cf. $\S 7,8$ below). We shall explain in the following that Problem $\left(A_{2}\right)$ is also reduced to the case of simple Lie algebras.

§7. Reduction of the Problem $(A)$ to the reductive case

Let $g$ be a Lie algebra over $R$ and $\mathfrak{r}$ the radical of $g$. If $(d, E)$ is a completely reducible real representation of $g$ over the finite dimensional real vector space $E$, then, as is well-known," every element of the ideal $[\mathfrak{r}, \mathfrak{g}]$ is mapped by $d$ to zero. Thus every completely reducible representation of $g$ is that of $g /[\mathfrak{r}, g]$. Now $\bar{g}=g /[\mathfrak{r}, g]$ is a reductive Lie algebra, i.e. the radical $\overline{\mathfrak{r}}$ $=\mathfrak{r} /[\mathfrak{r}, \mathfrak{g}]$ of $\overrightarrow{\mathfrak{g}}$ coincides with the center of $\overline{\mathfrak{g}}$. Hence we may assume without loss of generality, in dealing with the Problem $A$, that $g$ is a reductive Lie algebra. Let $z$ be the center of $g$. Then a representation $(d, E)$ of $g$ is a completely reducible representation of $g$, if and only if for every element $Z \in \delta$, $d(Z)$ is a semi-simple linear operator of $E^{3)}$

Now let $a$ be any ideal of a reductive Lie algebra $g$. Then since there is an ideal $\mathfrak{b}$ of $g$ such that $\mathfrak{g}=\mathfrak{a}+\mathfrak{b}, \mathfrak{a} \cap \mathfrak{b}=(0)$, the center of $\mathfrak{a}$ is contained in the center $z$ of $\mathrm{g}$. Hence every completely reducible representation of $\mathrm{g}$ induces also a completely reducible representation of a.

\section{$\S 8$. Induced irreducible representations on ideals}

Let $g$ be a reductive Lie algebra over $R$ and $a$ be an ideal of $g$. Then there

2) cf. for example, C. Chevalley, Algebraic Lie Algebras, Ann. of Math. vol. 48 (1946).

3) cf. C. Chevalley, Théorie des groupes de Lie, III (1955), Chap. IV, $\S 4, n^{0} 1$. 
is an ideal $\mathfrak{b}$ of $\mathfrak{a}$ such that

$$
a=a+b, a \cap b=(0) .
$$

Now let $(\rho, V)$ be a completely reducible representation of $\mathfrak{g}$. Then $(\rho, V)$ induces a representation of a over $V$ which is also completely reducible (cf. $\S 7$ ). Hence $V$ can be decomposed into a direct sum of a-invariant subspaces:

$$
V=V_{1}+V_{2}+\ldots+V_{r}
$$

where every $V_{i}$ is a minimal a-invariant subspace, i.e. the representation of $a$ induced by $\rho$ on $V_{i}$ is irreducible. Let $B$ be any element in $\mathfrak{b}$. Let us consider a linear mapping $\varphi_{B}$ from $V_{i}$ into $V_{j}$ defined as follows: For $x \in V_{i}$, let $\varphi_{B}(x)$ be the $V_{j}$-component of $\rho(B) x$ i.e. if we write

$$
\rho(B) x=y_{1}+\ldots+y_{r}, y_{k} \in V_{k}(k=1, \ldots, r)
$$

then $\varphi_{B}(x)=y_{j} . \quad$ Since every $V_{k}$ is a-invariant, we have $\varphi_{B} \circ \rho(x)=\rho(X) \circ \varphi_{B}$ for every $X \in a$. Then, if $V_{i}$ and $V_{j}$ are not equivalent as the representation spaces of a we have $\varphi_{B}=0$ by Schur's lemma. In other words, let $V_{k_{1}}, \ldots, V_{k_{p}}$ be the system of all subspaces $V_{k}$ in (4) which is equivalent to $V_{i}$ as representation spaces of $\mathfrak{a}$, then $U=V_{k_{1}}+\ldots+V_{k_{p}}$ is b-invariant. Hence $U$ is also g-invariant. If $(\rho, V)$ is irreducible with respect to $\mathrm{g}$, then $U=V$. Thus we have the following lemma by means of Jordan-Hölder's theorem.

LemMa 2. Let $(\rho, V)$ be an irreducible representation of a reductive Lie algebra $\mathrm{g}$ and $\mathfrak{a}$ be an ideal of $\mathrm{g}$. Then every minimal a-invariant subspaces of $V$ are equivalent to each other as representation spaces of a with respect to the representation of a induced by $\rho$.

In this case we shall denote by $V_{\mathfrak{a}}$ one of the minimal $a$-invariant subspaces of $V$, and by $\rho_{\mathfrak{a}}$ the irreducible representation of $a$ induced by $\rho$ on $V_{\mathfrak{a}}$. The representation $\left(\rho_{\mathfrak{a}}, V_{\mathfrak{a}}\right)$ is determined up to an equivalence. We shall call this irreducible representation $\left(\rho_{\mathfrak{a}}, V_{\mathfrak{a}}\right)$ of a the induced irreducible representation of a by the irreducible representation $(\rho, V)$ of $g$.

Now let $(\rho, V)$ be an irreducible representation of $g$ and (4) be a decomposition of $V$ into a direct sum of irreducible a-invariant subspaces $V_{1}, \ldots, V_{r}$. We may take $V_{1}$ as $V_{\mathfrak{a}}$. Since $V_{1}$ and $V_{i}$ are equivalent, we can choose equivalence mappings $\varphi_{1}^{i}: \quad V_{1} \rightarrow V_{i}$ with $\varphi_{1}^{1}=$ identity. We put $\varphi_{1}^{i} \circ\left(\varphi_{1}^{j}\right)^{-1}=\varphi_{j}^{i}$, then $\varphi_{j}^{i}: V_{j} \rightarrow V_{i}$ is an equivalence mapping as representation spaces of $a$, 
Let us fix the system $\left\{\varphi_{j}^{i}\right\}$ of equivalence mappings. Note that $\varphi_{j}^{i} \circ \varphi_{k}^{j}$ $=c_{k}^{i}$. Now let $C^{r}$ be the Cartesian space with $r$ complex components. Let us construct a representation of $\mathfrak{b}$ on $C^{r}$. Let $B \in \mathfrak{b}$. Denoting by $\pi_{j}$ the projection from $V$ onto $V_{j}$ with respect to the decomposition (4), we have a linear endomorphism $\varphi_{j}^{i} \circ \pi_{j} \circ \rho(B)$ of $V$ which is commutative with every $\rho(X), X \in \mathfrak{a}$. Then, by Schur's lemma, $\varphi_{j}^{i} \circ \pi_{j}^{\circ} \rho(B)$ is a scalar operator on $V_{i}$. Denote this scalar by $\sigma_{j}^{i}(B)$, then we obtain $\rho(B) \varphi_{1}^{i}(x)=\sum_{j} \sigma_{j}^{i}(B) \varphi_{i}^{j} \varphi_{1}^{i}(x)$ for $x \in V_{1}$. Denote by $\sigma(B)$ the $r \times r$ matrix $\sigma(B)=\left(\sigma_{j}^{i}(B)\right) . \quad \sigma(B)$ is a linear endomorphism of $C^{r}$. Now $B \rightarrow \sigma(B)$ is a representation of $b$ on $C^{r}$. To show this, let us consider a bilinear mapping from $V_{\mathfrak{a}} \times C^{r}$ into $V$ defined as follows : let $x \in V_{\mathfrak{a}}$ $\left(=V_{1}\right), \lambda \in C^{r}$. Then we write $[x, \lambda]=\sum_{i=1}^{r} \lambda_{i} \varphi_{1}^{i}(x)$ where $\lambda=\left(\lambda_{1}, \ldots, \lambda_{r}\right) \in C^{r}$. Then $(x, \lambda) \rightarrow[x, \lambda]$ is a bilinear mapping $V_{\mathfrak{a}} \times C^{r} \rightarrow V$ and obviously any element of $V$ can be expressed as a finite sum of elements of a form $[x, \lambda]$, $x \in V_{\mathfrak{a}}, \lambda \in C^{r}$. Then we obtain an onto linear mapping $V_{\mathfrak{a}} \otimes C^{r} \rightarrow V$ such that $x \otimes \lambda \rightarrow[x, \lambda]$. Since $\operatorname{dim} V=\operatorname{dim}\left(V_{\mathfrak{a}} \otimes C^{r}\right)$, this linear mapping is a linear isomorphism of $V$ with $V_{\mathfrak{a}} \otimes C^{r}$. So we identify $V$ with $V_{\mathfrak{a}} \otimes C^{r}$ and write $x \otimes \lambda$ instead of $[x, \lambda]$. Now we have

$$
\rho(A)(x \otimes \lambda)=\sum \lambda_{i} \rho(A) \varphi_{1}^{i}(x)=\sum \lambda_{i} \varphi_{1}^{i}\left(\rho_{\mathfrak{a}}(A) x\right)=\rho_{\mathfrak{a}}(A) x \otimes \lambda
$$

for every $A \in \mathfrak{a}$, and by $\rho(B) \varphi_{1}^{i}(x)=\sum_{j} \sigma_{j}^{i}(B) \varphi_{i}^{j} \varphi_{1}^{i}(x)=\sum_{j} \sigma_{j}^{i}(B) \varphi_{1}^{j}(x)$, we have for any $B \in \mathfrak{b}$

$$
\rho(B)(x \otimes \lambda)=\sum_{i} \lambda_{i} \rho(B) \varphi_{1}^{i}(x)=\sum_{i j} \lambda_{i} \sigma_{j}^{i}(B) \varphi_{1}^{j}(x)=x \otimes \sigma(B) \lambda
$$

Then for $B_{1}, B_{2} \in \mathfrak{b}$ we obtain by

$$
\begin{aligned}
\rho\left(\left[B_{1}, B_{2}\right]\right)(x \otimes \lambda) & =\rho\left(B_{1}\right) \rho\left(B_{2}\right)(x \otimes \lambda)-\rho\left(B_{2}\right) \rho\left(B_{1}\right)(x \otimes \lambda) \\
& =x \otimes \sigma\left(B_{1}\right) \sigma\left(B_{2}\right) \lambda-x \otimes \sigma\left(B_{2}\right) \sigma\left(B_{1}\right) \lambda \\
& =x \otimes\left[\sigma\left(B_{1}\right), \sigma\left(B_{2}\right)\right] \lambda
\end{aligned}
$$

that $\sigma\left(\left[B_{1}, B_{2}\right]\right)=\left[\sigma\left(B_{1}\right), \sigma\left(B_{2}\right)\right]$ i.e., $B \rightarrow \sigma(B)$ is a representation of $\mathfrak{b}$ on $C^{r}$.

Now let $\pi_{\mathfrak{a}}$ and $\pi_{\mathfrak{b}}$ be the projections from $\mathfrak{g}$ onto $\mathfrak{a}$ and $\mathfrak{b}$ respectively with respect to the decomposition $\mathfrak{g}=\mathfrak{a}+\mathfrak{b}$. Then $\rho_{\mathfrak{a}}{ }^{\circ} \pi \mathfrak{a}$ and $\sigma^{\circ} \pi_{\mathfrak{b}}$ are representations of 9 . From (5), (6) we also see that the representation $(\rho, V)$ of $g$ is equivalent to the tensor product of two representation $\left(\rho_{\mathfrak{a}} \circ \pi_{\mathfrak{a}}, V_{\mathfrak{a}}\right),\left(\sigma^{\circ} \pi_{\mathfrak{b}}, C^{r}\right)$ : . 
$\rho=\rho_{\mathfrak{d}}{ }^{2} \pi \mathfrak{a} \circlearrowleft \sigma^{\circ} \pi_{\mathfrak{b}}{ }^{d,} V=V_{\mathfrak{a}} \otimes C^{r}$. . The representation $\left(\sigma, C^{r}\right)$ of $\mathfrak{b}$ is irreducible. In fact if $C^{r}$ contains a non-trivial g-invariant subspace $U$, then $V_{a} \otimes U$ is obviously a non-trivial g-invariant subspace of $V=V_{a} \otimes C^{r}$ by (5), (6).

Now let us show that $\left(\sigma, C^{r}\right)$ is equivalent to the induced irreducible representation of $b$ by the irreducible representation $(\rho, V)$ of $\mathrm{g}$. In fact, let $e_{1}, \ldots, e_{i}$ be a base of $V_{a}$. Then $V=\sum_{i} e_{i} \otimes C^{r}$ is a direct sum of $\mathfrak{b}$-invariant subspaces $e_{i} \otimes C^{r}$, and since every $e_{i} \otimes C^{r}$ is b-irreducible, $e_{i} \otimes C^{r}$ is a minimal $b$-invariant subspace of $V$. Hence $\rho_{\mathfrak{E}} \sim \sigma$ by $(6)$. Thus we have the following

Lemma 3. Let $\mathfrak{g}$ be a reductive Lie algebra over $R$ and $\mathfrak{g}=\mathfrak{a}+\mathfrak{b}$ be $a$ decomposition of $\mathfrak{g}$ into ideals $\mathfrak{a}, \mathfrak{b}$ of $\mathrm{g}$. Then every irreducible representation $(\rho, V)$ of $\mathfrak{g}$ is equivalent to the tensor product of two irreducible representations $\left(\rho_{\mathfrak{a}} \circ \pi_{\mathfrak{a}}, V_{\mathfrak{a}}\right)$ and $\left(\rho_{\mathfrak{b}}^{\circ} \pi_{\mathfrak{v}}, V_{\mathfrak{b}}\right)$, where $\pi_{\mathfrak{a}}$ and $\pi_{\mathfrak{b}}$ are projections of $\mathfrak{a}$ onto a and $\mathfrak{b}$ respectively.

Conversely, if $\left(\rho_{1}, U_{1}\right)$ and $\left(\rho_{2}, U_{2}\right)$ are arbitrary irreducible representations of $\mathfrak{a}$ and $\mathfrak{b}$ respectively, then $\left(\rho_{1} \circ \pi_{\mathfrak{a}} \rho_{2} \circ \pi_{\mathfrak{b}}, \quad U_{1}\left(\dot{x}, U_{\mathfrak{2}}\right)\right.$ is an irreducible representation of g. To show this, let $e_{1}, \ldots, e_{r}$ be any base of $U_{2}\left(r=\operatorname{dim} U_{2}\right)$. Then we have $U_{1} \otimes U_{2}=\sum_{j} U_{1} \otimes e_{j}$ (direct sum), and every $U_{1} \otimes e_{j}$ is an $a$ invariant subspace of $U_{1} \otimes U_{2}$ which is a-irreducible. Then by Jordan-Hölder's theorem, every minimal a-invariant subspace of $U_{1} U_{2}$ are equivalent to each other and are equivalent to $U_{1}$. Analogously, every minimal b-invariant subspace of $U_{1} \otimes U_{2}$ are equivalent to each other and are equivalent to $U_{2}$. Now let $V$ be any minimal g-invariant subspace of $U_{1}(x) U_{2}$, and let $\rho$ be the irreducible representation of $\Omega$ induced by $\rho_{1} \circ \pi_{a} \rho_{2} \circ \pi_{b}$ on $V$. Then, from what we remarked above, we have $\rho_{\mathfrak{a}} \sim \rho_{1}, \rho_{\mathfrak{b}} \sim \rho_{\mathfrak{2}}$. Then $\operatorname{dim} V=\operatorname{dim} U_{1} \cdot \operatorname{dim}$ $U_{2}$. Hence $V=U_{1} \otimes U_{2}$. Thus $U_{1} \otimes U_{2}$ is irreducible.

Thus in order to find all irreducible representation of $\mathfrak{g}$, it is sufficient to find all irreducible representations of $\mathfrak{a}$ and $\mathfrak{b}$. We note here that for two

4) In general, the tensor product of two representations $\left(\rho_{1}, V_{1}\right),\left(\rho_{2}, V_{2}\right)$ of a Lie algebra $g$ is defined as the following representation $(\rho, V)$ of $g$ : the representation space $V$ is the tensor product of $V_{1}, V_{2}$, i.e. $V=V_{1} \otimes V_{2}$, and for $X \in \mathfrak{B}, \rho(X)$ is an endomorphism of $V$ given by

$$
\rho(X)=\rho_{1}(X) \otimes I_{2}+I_{1} \otimes \rho_{2}(X) \text {, i.e. } \rho(X)(x \otimes y)=\rho_{1}(X) x \otimes y+x \otimes \rho_{2}(X) y,
$$

where $I_{1}, I_{2}$ denote the identical operators of $V_{1}, V_{2}$ respectively. This representation $\rho$ is denoted by $\rho=\rho_{1}-\rho_{2}$ ( $\rho$ is also called the tensor sum of $\rho_{1}, \rho_{2}$ ). 
irreducible representations $(\rho, V),(\sigma, U)$ of $\mathfrak{g}$, we have $\rho \sim \sigma$ if and only if $\rho_{\mathfrak{a}} \sim \sigma_{\mathfrak{a}}$ and $\rho_{\mathfrak{E}} \sim \sigma_{\mathfrak{b}}$. These facts are easily extended to the case of the decomposition of $\mathfrak{g}$ into many ideals $: \mathfrak{g}=\mathfrak{a}+\mathfrak{b}+\ldots+c$. If we take in particular the decomposition of 3 into simple ideals:

$$
\mathfrak{n}=g_{1}+g_{2}+\ldots+g_{r},
$$

then, Problem $\left(A_{1}\right)$ is reduced to the case of simple Lie algebras.

\section{$\S 9$. Criterions of self-conjugateness}

Let $(\rho, V)$ be an irreducible representation of a (reductive) Lie algebra $\mathfrak{g}$ over $R$. Let us consider the condition for $\rho$ to be self-conjugate. If $\rho \sim \bar{\rho}$, then there exists an anti-linear automorphism $J$ of $V$ such that $J \circ \rho(X)=\rho(X) \circ J$ for every $X \in g(\mathrm{cf} . \S 4)$. Then $J^{2}$ is a linear automorphism of $V$ which is commutative with every $\rho(X), X \in g$. Then by Schur's lemma, $J^{2}$ is a scalar operator of $V: J^{2}=c I(c \in C)$. Now let us call (after E. Cartan) an anti-linear automorphism $J$ of a complex vector space $V$ an anti-involution if $J^{2}$ is a scalar operator of $V$. If $J$ is an anti-involution of $V$ and $J^{2}=c I$, then $c$ is a real number. In fact, let $e_{1}, \ldots, e_{n}$ be any base of $V$. Then, putting $J e_{i}=\sum_{j} \alpha_{i}^{j} e_{j}$ $\left(\alpha_{i}^{j} \in C\right)$, we have $J^{2} e_{i}=\sum_{j, k} \bar{\alpha}_{i}^{j} \alpha_{j}^{k} e_{k}$. Hence, if we denote by $A$ the complex matrix $\left(\alpha_{i}^{j}\right)$, we have

$$
A \bar{A}=c I .
$$

Then by $c \neq 0$, we have $A \bar{A}=\bar{A} A$ and so $c=\bar{c}$. Hence $c$ is real. If $c>0(c<0)$ then $J$ is called an anti-involution of the first (second) kind. We also say that the index of $J$ is $+1(-1)$ if $J$ is an anti-involution of the first (second) kind. We remark that if $J$ is an anti-involution of index $\varepsilon(\varepsilon= \pm 1)$, then for any complex number $\gamma \neq 0, \gamma J$ is also an anti-involution of index $\varepsilon$ (Note that $\left.(\gamma J)^{2}=|\gamma|^{2} J^{2}\right)$.

We have seen in the above that if $(\rho, V)$ is a self-conjugate, irreducible representation, then there is an anti-involution $J$ which is invariant by $\rho$. Now let us note that such an anti-involution is unique up to scalar multiples. In fact, if $J$ and $J^{\prime}$ are invariant anti-involutions, then $J^{\prime} J^{-1}$ is a linear automorphism of $V$ which is commutative with every $\rho(X), X \in 8$. Hence $J^{\prime}=\gamma J$ for some $r \in C$ by Shur's lemma. Thus the index of $J$ is independent on the choice of $J$. This index is called the index of a self conjugate, irreducible representation 
$(\rho, V)$.

LËмma 4. Let $(\rho, V)$ be an irreducible representation of 8 . Then $\rho$ is of the first class if and only if $\rho$ is self-conjugate and of index 1.

Proof. Let $(\rho, V)$ be of the first class. Then $V_{R}$ contains a $\rho_{R}$-invariant (real) subspace $E$ such that

$$
V=E+\sqrt{-1} E, E \cap \sqrt{ }-1 E=(0) .
$$

Let $J$ be the complex conjugate operation of $V$ with respect to $E: J(x+\sqrt{ }-1 y)$ $=x-\sqrt{-1} y(x, y \in E)$. Then $J^{2}=I$ and $J$ is invariant by $\rho$ since $E$ is $\rho_{R^{-}}$ invariant.

Conversely let $\rho$ be self-conjugate and of index 1 . Then there is an antiinvolution $J$ of $V$ which is invariant by $\rho$ and $J^{2}=I$. Let $E=\{x \in V ; J x=x\}$. Then $E$ is a real subspace, i.e. $E$ is a subspace of $V_{R}$ and moreover $E$ is invariant by $\rho_{R}$. Now every element $x \in V$ can be expressed as $x=\frac{1}{2}(x+J x)$ $+\frac{1}{2}(x-J x)$, where we have $x+J x \in E$ and $x-J x \in\{x \in V ; J x=-x\}=\sqrt{-1} E$. Thus we have $V=E+\sqrt{-1} E, E \cap \sqrt{ }-1 E=(0)$. Then $E$ is a non-trivial $\rho_{R^{-}}$ invariant subspace of $V_{R}$. Thus $\left(\rho_{R}, V_{R}\right)$ is reducible and $\rho$ is of the first class. Thus lemma 4 is proved.

Thus Problem $\left(A_{2}\right)$ is reduced to decide the self-conjugateness and the index of an irreducible representation. We note here a necessary condition for a representation $(\rho, V)$ to be self-conjugate.

Lemma 5. If a representation ( $\rho, V)$ of a real Lie algebra 8 is selfconjugate, then $\rho(g) \cap \sqrt{-1} \rho(g)=(0)$.

Proof. Let $J$ be a anti-linear automorphism of $V$ which is invariant by $\rho$. If $\rho(A)=\sqrt{-1} \rho(B) \in \rho(\mathfrak{g}) \cap \sqrt{ }-1 \rho(\mathfrak{g}),(A, B \in \mathfrak{g})$, then we have $J \rho(A) J^{-1}$ $=\rho(A), J(\sqrt{-1} \rho(B)) J^{-1}=\sqrt{ }-1 \rho(B)$. On the other hand, $J(\sqrt{-1} \rho(B)) J^{-1}$ $=-\sqrt{-1} J \rho(B) J^{-1}=-\sqrt{-1} \rho(B)$ Therefore we have $\rho(A)=\sqrt{-1} \rho(B)=0$ and $\rho(\mathfrak{g}) \cap \sqrt{-1} \rho(g)=(0)$, Q.E.D.

Now let $g$ be a reductive Lie algebra over $R$ and let

$$
\mathfrak{g}=\mathfrak{g}_{1}+g_{2}+\ldots+\mathfrak{g}_{r}
$$

be the decomposition of $g$ into simple ideals $g_{1}, \ldots, g_{r}$. We shall denote by $\pi_{i}$ the projection of $g$ onto $g_{i}$ with respect to the above decomposition. Let 
$(\rho, V)$ be an irreducible representation of $g$ and $\rho_{i}(i=1, \ldots, r)$ be the induced irreducible representations of $g_{i}$ by the irreducible representation $\rho$ of $g$. Under these notations we have the following

Lemma 6. $\rho \sim \bar{\rho}$ if and only if $\rho_{i} \sim \bar{\rho}_{i}$ for $i=1, \ldots, r$. In this case the index $\varepsilon$ of $\rho$ is given by $\varepsilon=\varepsilon_{1} \varepsilon_{2} \ldots \varepsilon_{r}$ where $\varepsilon_{i}$ is the index of $\rho_{i}(i=1, \ldots, r)$.

Proof. Assume $\rho \sim \bar{\rho}$. Then there is an anti-involution $J$ which is invariant by $\rho$. Let $V_{1}$ be a minimal $g_{1}$-invariant subspace of $V$. Then $J V_{1}$ is also a minimal $g_{1}$-invariant subspace of $V$ as is seen easily. Then $V_{1}$ and $J V_{1}$ are equivalent as representation spaces of $g_{1}$ by lemma 2 . Hence we have $\rho_{1} \sim \bar{\rho}_{1}$. Analogously $\rho_{i} \sim \bar{\rho}_{i}(i=1, \ldots, r)$. Conversely assume that $\rho_{i} \sim \bar{\rho}_{i}(i=1, \ldots$, $r)$. Let $V_{1}, \ldots, V_{r}$ be the representation spaces of $\rho_{1}, \ldots, \rho_{r}$ respectively. Then we may assume that $V=V_{1} \otimes \ldots \otimes V_{r}$ and $\rho=\rho_{1} \circ \pi_{1} \oplus \ldots \oplus \rho_{r} \circ \pi_{r}$. Let $J_{i}(i=1, \ldots, r)$ be an anti-involution of $V_{i}$ which is invariant by $\rho_{i}$ and $J_{i}^{2}=\varepsilon_{i} I$. Then $J=J_{1} \otimes \ldots \otimes J_{r}$ is an anti-involution of $V$ and $J^{2}=J_{1}^{2} \otimes \ldots \otimes J_{r}^{2}$ $=\varepsilon_{1} \ldots \varepsilon_{r} I$. Moreover $J$ is invariant by $\rho$, since for $X=X_{1}+\ldots+X_{r} \in g$ $\left(X_{i} \in g_{i}, i=1, \ldots, r\right)$ we have $J \rho(X)=\left(J_{1} \otimes \ldots \otimes J_{r}\right)\left(\rho_{1}\left(X_{1}\right) \otimes I \otimes \ldots \otimes I\right.$ $\left.+\ldots+I \otimes \ldots \otimes I \otimes \rho_{r}\left(X_{r}\right)\right)=\rho(X) J$. Thus we have completed the proof.

Thus Problem $\left(A_{2}\right)$ is reduced to the case of real simple Lie algebras by lemmas 4,6 . In the following we shall consider this case.

\section{$\S 10$. Irreducible representation of real simple Lie algebras}

Let $g$ be a real simple Lie algebra. Then the following three cases are possible :

a) $g$ is 1-dimensional abelian Lie algebra,

b) $g$ is simple, non-abelian Lie algebra and $g^{C}$ is not simple,

c) $g$ is simple, non-abelian Lie algebra and $g^{c}$ is simple.

Let $g$ be an abelian Lie algebra of dimension 1 . Then an irreducible representation $(\rho, V)$ of $g$ is of degree 1 . Obviously $\rho$ is self-conjugate if and only if every element of $\rho(g)$ is a real multiple of the identity. Moreover, if $\rho \sim \bar{\rho}$ then clearly the index of $\rho$ is equal to 1 . Next let us consider the cases $b$ ), c) simultaneously. For this purpose we consider a real semi-simple Lie algebra 9 . Let $\mathfrak{h}$ be a Cartan subalgebra of $g$. "We denote by $l$ the dimension of $\mathfrak{h}$. Then $\mathfrak{h}^{c}$ is a Cartan subalgebra of $\mathfrak{g}^{c}$. We denote by $Z \rightarrow \bar{Z}$ the complex 
conjugate operation of $g^{c}$ with 'respect to $g$. Then we have $\alpha X+\beta Y=\bar{\alpha} \bar{X}$ $+\bar{\beta} \bar{Y}$ and $[\bar{X}, \bar{Y}]=[\bar{X}, \bar{Y}]$ for every $X, Y \in g^{c}, \alpha, \beta \in C$. Let $\Delta \ni \alpha, \beta$, $\ldots$, be the root system of $g^{c}$ with respect to the Cartan subalgebra $\mathfrak{h}^{c}$. Let $A$ be a linear form on $\mathfrak{h}^{\prime}$, then we denote by $\bar{A}$ the linear form on $\mathfrak{h}^{\prime}$ defined by $\bar{\Lambda}(H)=\bar{A}(\bar{H})$ for every $H \in \mathfrak{h}^{\prime}$. Then the mapping $A \rightarrow \bar{A}$ is an anti-linear involution of the dual vector space $\left(\mathfrak{h}^{c}\right)^{*}$ of $\mathfrak{h}^{c}$. We have clearly $\overline{\bar{\Lambda}}=\Lambda$

Lemma 7. $\bar{A}=\Delta$, i.e. the mapping $A \rightarrow \bar{A}$ induces a permutation of $\Delta$.

Proof. For $\alpha \in \Delta$, take a root vector $E_{\alpha} \neq 0$ in $g^{r}$. Then $\left[H, E_{\alpha}\right]=\alpha(H) E_{\alpha}$ for every $H \in \mathfrak{h}^{c}$. Hence we have $\left[\bar{H}, \bar{E}_{\alpha}\right]=\overline{\alpha(H)} \bar{E}_{\alpha}$, i.e. $\left[H, \bar{E}_{\alpha}\right]=\bar{\alpha}(H) \bar{E}_{\alpha}$. Consequently we have $\bar{\alpha} \in \Delta$, Q.E.D.

Let $R_{l}$ be the real subspace of $\left(\mathfrak{h}^{\prime}\right)^{*}$ consisting of all linear combinations of roots with real coefficients. Then the canonical inner product ${ }^{5)}\left(A_{1}, A_{2}\right)$ on $\left(\mathfrak{h}^{r}\right)^{*}$ is positive definite on $R_{l}$, and $R_{l}$ is an Euclidean space with respect to this inner product $\left(A_{1}, A_{2}\right)$. The anti-linear involution $A \rightarrow \bar{A}$ leaves $R_{l}$ invariant. Then by lemma 7 , we have ${ }^{6)}$

$$
\left(A_{1}, A_{2}\right)=\left(\bar{A}_{1}, \bar{A}_{2}\right)
$$

for every $d_{1}, d_{2} \in R_{l}$.

Let $I=\left\{\alpha_{1}, \ldots, \alpha_{l}\right\}$ be a fundamental root system ${ }^{i)}$ in $\Delta$. Then by lemma $7, \bar{\Pi}=\left\{\bar{\alpha}_{1}, \ldots, \bar{\alpha}_{l}\right\}$ is also a fundamental root system in $\Delta$. Hence there is an element $S_{0}$ in the Weyl group $\mathbf{W}$ of $\mathfrak{g}^{\prime}$ with respect to $\mathfrak{h}^{r}$ such that $S_{0}(\Pi)=\bar{\Pi}^{8)}$.

Now let $(\rho, V)$ be a representation of $g$. Then $\rho$ can be uniquely extended to a representation of $g^{c}$ on $V$ which we also denote by $(\rho, V)$. Let $A$ be a weight of $g^{\prime \prime}$ (with respect to $\mathfrak{h}^{\prime \prime}$ ) in the representation $(\rho, V)$. Then $\bar{A}$ is a weight of $g^{r}$ in the representation $(\bar{\rho}, \bar{V})$. In fact, let $x \neq 0$ a vector in $V$ such that $\rho(H) x=\Lambda(H) x$ for every $H \in \mathfrak{h}^{c}$. Then $\overline{\rho(H)} \bar{x}=\Lambda(H) \bar{x}$. Now we have

5) Let $\phi$ be the Killing form of $g^{c}$. Then for any $A \in\left(\mathfrak{h}^{c}\right)^{*}$, there coresponds uniquely an element $H_{\Lambda} \in \mathfrak{h}^{C}$ such that $\varphi\left(H_{\Lambda}, H\right)=\Lambda(H)$ for every $H$ in $\mathfrak{h}^{c}$. Then the canonical inner product of $\Lambda_{1}, \Lambda_{2} \in\left(\mathfrak{h}^{C}\right)^{*}$ is given by $\left(\Lambda_{1}, \Lambda_{2}\right)=\Phi\left(H_{\Lambda_{1}}, H_{\Lambda_{2}}\right)$.

6) cf. [2], Exposé $n^{0} 11$ et 12, Théorème 1.

7) I.e. every $\alpha \in \Delta$ is expressible uniquely as $\alpha=\sum m_{i} \alpha_{i}$ with integral coefficients $m_{i}$ such that $m_{1} \gg 0, \ldots, m_{l} \gg 0$ or $m_{1} \leqq 0, \ldots, m_{l} \leqq 0$.

8) cf. [2], Exposś $n^{0} 16$, Théorème 1. 
$\bar{\rho}(H)=\bar{\rho} \overline{(\bar{H}})$, because if $H=H_{1}+\sqrt{-1} H_{2}\left(H_{1}, H_{2} \in \mathfrak{h}\right)$, then $\bar{\rho}(H)=\bar{\rho}\left(H_{1}\right)$ $+\sqrt{-1} \bar{\rho}\left(H_{2}\right)=\overline{\rho\left(H_{1}\right)}+\sqrt{-1} \rho\left(H_{2}\right)=\overline{\rho\left(H_{1}-\sqrt{-1} H_{2}\right)}$. Hence $\bar{\rho}(H) \bar{x}=\bar{\Lambda}(H) \bar{x}$ for every $H \in \mathfrak{h}^{c}$. Consequently $\bar{A}$ is a weight of $g^{c}$ in the representation $(\bar{\rho}, \bar{V})$.

Thus, if we denote by $W(\rho)$ the set of all weights in the representation $(\rho, V)$ then we have

$$
W(\bar{\rho})=W(\bar{\rho}) .
$$

A weight $A$ in the representation $(\rho, V)$ is called extreme if we have for any root $\alpha, A+\alpha \notin W(\rho)$ or $A-\alpha \notin W(\rho)$. Then we have by (8) and lemma 7 the following

LEMMA 8. If $A$ is an extreme weight in $(\rho, V)$, then $\bar{A}$ is an extreme weight in $(\bar{\rho}, \bar{V})$.

Now let us introduce a lexicographical linear order in $R_{l}$ such that $\Pi$ becomes the set of all simple roots ${ }^{9)}$ in $\Delta$ with respect to this linear order. Then we can speak of the highest weight in the representation $(\rho, V)$. The following lemma is well-known.

Lemma 9. If $A_{0}$ is the highest weight in $(\rho, V)$ and $A_{1}$ is an extreme weight in the irreducible representation $(\rho, V)$. Then there is an element $S$ in the Weyl group $W$ such that $S\left(\Lambda_{1}\right)=\Lambda_{0}$.

Proof. Let $\Lambda_{2}$ be the highest weight among the set of weights $\left\{S\left(\Lambda_{1}\right)\right.$; $S \in W\}$. Replacing $\Lambda_{1}$ by $\Lambda_{2}$ if necessary, we may assume that $\Lambda_{1}=\Lambda_{2}$. Then we have $S_{\alpha}\left(A_{1}\right)=\Lambda_{1}-\frac{2\left(A_{1}, \alpha\right)}{(\alpha, \alpha)} \alpha \leqq \Lambda_{1}$. Hence we have $A_{1}-\alpha \in W(\rho)$ for every positive root $\alpha$ such that $\left(A_{1}, \alpha\right) \neq 0$, then $A_{1}+\alpha$ is not a weight in $(\rho, V)$. In other words, if we denote by $E_{\alpha}$ a root vector belonging to the root $\alpha$, then we have $\rho\left(E_{\alpha}\right) V_{\Lambda_{1}}=(0)$ for $\alpha>0$, where $V_{\Lambda_{1}}=\left\{x \in V ; \rho(H) x=\Lambda_{1}(H) x\right.$ for every $\left.H \in \mathfrak{G}^{c}\right\}$. Then easy induction shows that every subspace of the following form

9) A simple root is a positive root which not expressible as a sum of two positive roots. cf. [2], Exposé $n^{0} 10$. Now, a lexicographical linear order in $R_{l}$ is defined as follows: let $\xi=\sum \xi_{i} \alpha_{i}, \eta=\sum \eta_{i} \alpha_{i}$ be in $R_{l}$. Then we dęfine $\xi>\eta$ if $\xi_{1}=\eta_{1}, \ldots, i_{r-1}=\eta_{r-1}$, $\xi_{r}>\eta_{r}$ for some $r, 1 \leqq r \leqq l$. Then the set of all simple roots in $\Delta$ with respect to this linear order coincides with $\alpha_{1}, \ldots, \alpha l$. 


$$
V_{\Lambda_{1}}, \rho\left(E_{\beta_{1}}\right) \ldots \rho\left(E_{\beta_{1}}\right) V_{\Lambda_{1}},\left(\beta_{i} \in \Delta, i=1, \ldots, t\right)
$$

coincides with a subspace of the following form

$$
V_{\Lambda_{1}}, \rho\left(E_{\Upsilon_{1}}\right) \ldots \rho\left(E_{\Upsilon_{s}}\right) V_{\Lambda_{1}},\left(\gamma_{j} \in A, j=1, \ldots, s, \gamma_{j}<0\right) .
$$

Then by virtue of the irreducibility of $V$, we see that

$$
V=V_{\Lambda_{1}}+\sum_{\beta_{i} \in \Delta} \rho\left(E_{\beta_{1}}\right) \ldots \rho\left(E_{\beta_{l}}\right) V_{\Lambda_{1}}=V_{\Lambda_{1}}+\sum_{r_{j} \in \Delta, r_{\jmath}<0} \rho\left(E_{r_{1}}\right) \ldots \rho\left(E_{r_{s}}\right) V_{\Lambda_{1}} .
$$

Thus, $A_{1}$ is the highest weight in $(\rho, V)$, Q.E.D.

Now let $A_{1}, \ldots, A_{l}$ be the fundamental weight system of $g^{\prime \prime}$ determined by $\Pi$, i.e. $A_{1}, \ldots, A_{l}$ be the elements in $R_{l}$ such that

$$
\left(\Lambda_{i}, \frac{2 \alpha_{j}}{\left(\alpha_{i}, \alpha_{i}\right)}\right)=\delta_{i j}, \quad(1 \leqq i, j \leqq l) .
$$

Then, by (7), $\bar{A}_{1}, \ldots, \bar{A}_{l}$ are the fundamental weight system of $g^{\prime \prime}$ determined by $\bar{\Pi}$. On the other hand, since $S_{0}(I I)=\bar{\Pi}$, we have $S_{0}\left\{A_{1}, \ldots, A_{l}\right\}=\left\{\bar{A}_{1}, \ldots\right.$, $\left.\bar{A}_{l}\right\}$. i.e. $S_{0}\left(A_{i}\right)=\bar{A}_{r(i)}(i=1, \ldots, l)$ for some permutation $\sigma$ of $\{1, \ldots, l\}$.

Now let $\left(\rho_{i}, V_{i}\right)(i=1, \ldots, l)$ be the irreducible representation which has $A_{l}$ as the highest weight. $\rho_{1}, \ldots, \rho_{l}$ are called the fundamental representations determined by $\Pi$. Then the highest weight $.1_{i}^{\prime}$ of the irreducible representation $\bar{\rho}_{i}$ is expressible in the form $A_{i}^{\prime}=S\left(\bar{A}_{i}\right)$, where $S$ is an element in the Weyl group by lemmas 8,9 . Then we have

$$
A_{i}^{\prime}=S_{0}\left(A_{\sigma-1(i)}\right) \text {. }
$$

Then we have $A_{i}^{\prime}=A_{\sigma^{-1}(i)}$ since $A_{i}^{\prime}$ and $A_{\sigma^{-1}(i)}$ are both dominant weights. ${ }^{10)}$ Consequently, we have

$$
\bar{\rho}_{i} \sim \rho_{s-1(i)}(i=1, \ldots, l)
$$

Now we see that $\sigma^{2}=1$ by (9). Then arranging the order $\alpha_{1}, \ldots, \alpha_{l}$ if necessary, we may and shall assume that $\sigma(1)=2, \sigma(3)=4, \ldots, \sigma(2 k-1)$ $=2 k, \sigma(2 k+1)=2 k+1, \ldots, \sigma(l)=l$.

Let $(\rho, V)$ be an irreducible representation and $A$ be the highest weight of $\rho$. Then we have

$$
A=m_{1} A_{1}+\ldots+m_{l} d_{l}
$$

where $m_{1}, \ldots, m_{l}$ are non-negative integers. Then we have $. \bar{l}=\Sigma m_{i} \overline{1}_{i}$

10) A weight $\alpha$ is called dominant if $S A \leqq A$ for any element $S$ in the Weyl group $\|$. 
$=S_{0}\left(\Sigma m_{i} 1_{\sigma^{-1}(i)}\right)$. Consequently $\Sigma m_{i} \Lambda_{\sigma^{-1}(i)}$ is conjugate to the highest weight $A^{\prime}$ of $\bar{\rho}$ under the Weyl group. On the other hand, since $\Sigma m_{i} A_{\sigma^{-1}(i)}$ is a dominant weight, $\Sigma m_{i} \Lambda_{\sigma^{-1}(i)}$ must coincide with $\Lambda^{\prime}: A^{\prime}=\Sigma m_{i} \Lambda_{\sigma-1(i)}=\Sigma m_{\sigma(i ;} A_{i}$.

Then we have $\rho \sim \bar{\rho}$. if and only if $A=\Lambda^{\prime}$, in other words, we have $\rho \sim \bar{\rho}$ if and only if

$$
m_{1}=m_{2}, m_{3}=m_{1}, \ldots, m_{2 k-1}=m_{2 k} .
$$

Now let us consider the index $\varepsilon$ of $\rho$ when $\rho \sim \bar{\rho}$. Let $\varepsilon_{2 k+j}$ be the index of $\rho_{2 k+j}(j=1, \ldots, l-2 k)$. We assert that

$$
\varepsilon=\varepsilon_{2 k+1}^{m_{2 k+1}} \ldots \varepsilon_{l}^{m_{l}} \text {. }
$$

To prove (11), we shall recall the definition of the Cartan composite:

Let $(\rho, V),(\sigma, U)$ be two irreducible representations of $g$. Let $\Lambda, \Lambda^{\prime}$ be the highest weight of $\rho, \sigma$ respectively. Let $W$ be the minimal invariant subspace of $V \otimes U$ generated by $V_{\Lambda} \otimes U_{\Lambda^{\prime}},{ }^{11)}$ and $\tau$ be the induced irreducible representation by $\rho \oplus \sigma$ on $W$. Then the irreducible representation $(\tau, W)$ is called the Cartan composite of $\rho$ and $\sigma$, which we denote by $\tau=\rho * \sigma, W=V * U$. Then the highest weight of $\tau$ is $A+\Lambda^{\prime}$. The operation $*$ is associative and $\rho * \sigma \sim \sigma * \rho$. By the criterion (10), if $\rho \sim \bar{\rho}$ and $\sigma \sim \bar{\sigma}$ then we have $\tau \sim \bar{\tau}$. Now

LEMMA 10. Let $(\rho, V)(\sigma, U)$ be irreducible, self-conjugate representations of indices $\varepsilon, \varepsilon^{\prime}$ respectively. Then the index of $\tau=\rho * \sigma$ is $\varepsilon \varepsilon^{\prime}$.

Proof. Let $J, J^{\prime}$ be the anti-involutions on $V, U$ which are invariant by $\rho, \sigma$ respectively and $J^{2}=\varepsilon I, J^{\prime 2}=\varepsilon^{\prime} I$. Then $J \otimes J^{\prime}$ is an anti-involution on $V \otimes U$ invariant by $\rho \otimes \sigma$. We have $\left(J \otimes J^{\prime}\right)^{2}=\varepsilon \varepsilon^{\prime} \cdot I$. Now put $W=V * U$ and decompose $V \otimes U$ into the direct sum of irreducible subspaces:

$$
V \otimes U=W_{1}+\ldots+W_{r}, \quad\left(W_{1}=W\right) .
$$

Let us denote by $\pi_{i}$ the projection from $V \otimes U$ onto $W_{i}$ with respect to the above decomposition. Then $\varphi_{i}=\pi_{i} \circ\left(J \otimes J^{\prime}\right)$ is an anti-linear mapping from $W_{1}$ into $W_{i}$, and we have

$$
\varphi_{i} \circ \tau(X)=\tau(X) \circ \varphi_{i} \quad \text { for every } X \in g .
$$

Since every $W_{i}$ is irreducible, we have then, $\bar{W}_{1} \sim W_{i}$ if $\varphi_{i} \neq 0$. However we

11) $V_{\Lambda}$ and $U_{\Lambda^{\prime}}$ mean the eigen-spaces of $\Lambda, \Lambda^{\prime}$ respectively. 
have $\tilde{W}_{1} \sim W_{1}+W_{i}$ for every $i:>1$, hence we must have $\varphi_{i}=0$ for every $i>1$. In other words, $\left(J \dot{x} J^{\prime}\right)\left(W_{1}\right)=W_{1}$. Thus, $J \otimes J^{\prime}$ induces an anti-involution on $W_{1}$ of index $\varepsilon \varepsilon^{\prime} . \quad J \otimes J^{\prime}$ is clearly invariant by $\tau$, Q.E.D.

Lemma 11. Let $(\rho, V)$ be any irreducible representation of 8 . Then $\rho+\bar{\rho}$ is self-conjugate and of index 1 .

Proof. Let $J: V \otimes \bar{V} \rightarrow V \otimes \bar{V}$ be a mapping defined by

$$
J(x \otimes \bar{y})=y \otimes \bar{x}, \quad(x, y \in V) .
$$

Then $J$ is an anti-involution of index 1. $J$ is invariant by $\rho \oplus \bar{\rho}$ :

$$
\begin{gathered}
(\rho \oplus \bar{\rho})(X) \circ J(x \otimes \bar{y})=\rho(X) y \otimes \bar{x}+y \otimes \bar{\rho}(X) \bar{x}=J(x \otimes \bar{\rho}(X) \bar{y}+\rho(X) x \otimes \bar{y}) \\
=J \circ(\rho \oplus \bar{\rho})(X)(x \otimes \bar{y}) .
\end{gathered}
$$

Now let $A=\Sigma m_{i} A_{i}$ be the highest weight of $\rho$. Then the highest weight of $\rho * \bar{\rho}$ is given by $\left(m_{1}+m_{2}\right) A_{1}+\left(m_{1}+m_{2}\right) A_{2}+\ldots+\left(m_{2 k-1}+m_{2 k}\right) A_{2 k}+2 m_{2 k+1} A_{2 k+1}+$ $\ldots+2 m_{l} \Lambda_{l}$. Hence $\rho * \bar{\rho}$ is self-conjugate by the criterion (10). Then analogously as in the proof of lemma 10, we have $J(V * \vec{V})=V * \vec{V}$. Thus $\rho * \rho$ is of index 1, Q.E.D.

Now let us prove (11). Let us express the highest $A$ of the irreducible representation $(\rho, V)$ as follows: $A=m_{1} A_{1}+\ldots+m_{l} A_{l}$. Then, we have

$$
\rho=\overbrace{\rho_{1} * \ldots * \rho_{1}}^{m_{1} \text {-times }} \ldots * \overbrace{\rho_{l} * \ldots * \rho_{l}}^{m_{l} \text {-times }}
$$

Consequently, by lemmas 10,11 , we have (11). (Note that $\bar{\rho}_{1} \sim \rho_{2}, \bar{\rho}_{3} \sim \rho_{1}$, $\left.\ldots, \bar{\rho}_{2 k-1} \sim \rho_{2 k}\right)$. Thus we have the following

ThEOREM 2. Let $\mathrm{g}$ be a real semi-simple Lie algebra and $\mathfrak{h}$ be a Cartan subalgebra of $\mathrm{g}$. Let $\alpha_{1}, \ldots, \alpha_{l}$ be any fundamental root system of $\mathfrak{g}^{\prime}$ with respect to the Catyan subalgebra $\mathfrak{h}^{\prime}$ of $\mathrm{g}^{t}$. Let $\mathrm{A}_{1}, \ldots, \mathrm{A}_{l}$ be the fundamental weights of $g^{\prime \prime}$ determined by $\alpha_{1}, \ldots, \alpha l$. Let $\mu_{1}, \ldots, \rho_{l}$ be the irreducible representations of $\Omega^{c}$ whose highest weights are $1_{1}, \ldots, 1_{l}$ respectively. (The linear order between weights is determined by $\left.\alpha_{1}, \ldots, \alpha_{l}\right)$.

(i) Then there is a permutation $\sigma$ of $1, \ldots, l$ such that

$$
\bar{\rho}_{\text {o(i) }} \sim \rho_{i} \quad(i=1, \ldots, l),
$$

and $\sigma^{2}=1$.

(ii) Let us arrange the order of $\alpha_{1}, \ldots, \alpha_{l}$ so that $\bar{\rho}_{1} \sim \rho_{2}, \bar{\rho}_{3} \sim \rho_{1}, \ldots, \rho_{2 k-1}$ 
$\sim \rho_{i k}, \bar{\rho}_{2 k+1} \sim \rho_{2 k+1}, \ldots, \bar{\rho}_{l} \sim \rho_{l}$ in $(i) . \quad$ Let $\varepsilon_{2 k+j}$ be the index of $\rho_{2 k+j}(j=1$, $\ldots, l-2 k)$. Let $(\rho, V)$ be an irreducible representation of $\mathrm{g}$ with the highest weight

$$
\Lambda=m_{1} A_{1}+m_{2} \Lambda_{2}+\ldots+m_{l} A_{l},
$$

then the highest weight of $\bar{\rho}$ is given by

$$
\begin{gathered}
m_{2} \Lambda_{1}+m_{1} \Lambda_{2}+m_{4} \Lambda_{3}+m_{3} \Lambda_{4}+\ldots+m_{2 k} \Lambda_{2 k-1}+m_{2 k-1} \Lambda_{2 k} \\
+m_{2 k+1} \Lambda_{2 k+1}+\ldots+m_{l} \Lambda_{l}
\end{gathered}
$$

and $\rho$ is self-conjugate if and only if

$$
m_{1}=m_{2}, m_{3}=m_{4}, \ldots, m_{2 k-1}=m_{2 k},
$$

and then the index $\varepsilon$ of $\rho$ is given by

$$
\varepsilon=\varepsilon_{2 k+1}^{m_{2 k+1}} \ldots \varepsilon_{l}^{m_{l}} \text {. }
$$

\section{§ 11. A Criterion in Case $(b)$}

As an application of Theorem 2, let us consider the case where $g$ is a real simple Lie algebra such that $g^{\prime}$ is not simple. In this case $g^{\prime}$ is a direct sum of two (complex) simple ideals ${ }^{12)}$ :

$$
g^{\prime \prime}=\mathfrak{a}+\overline{\mathfrak{a}}
$$

where bar means the complex conjugate operation of $g^{\prime \prime}$ with respect to 8 . Then the scalar restriction $\mathfrak{a}_{R}$ is isomorphic with $\mathfrak{g}$ under the mapping $x \rightarrow X$ $+\bar{X}\left(X \in \mathfrak{a}_{R}\right)$. Let $\mathfrak{b}$ be any Cartan subalgebra of $a$. Then $\mathfrak{h}=\{X+\bar{X} ; X \in \mathfrak{b}\}$ is a Cartan subalgebra of $\mathfrak{g}$ as is seen easily. Further $\bar{b}$ is a Cartan subalgebra of $\bar{a}$, and we have $\mathfrak{h}^{\prime}=\mathfrak{b}+\bar{b}$. Let $\Delta_{1}$ be the root system of $\mathfrak{a}$ with respect to $\mathfrak{b}$. Then every $\alpha \in \Delta_{1}$ is extended to a linear form on $\mathfrak{h}^{c}$ (which we also denote by $\alpha$ ) putting $\alpha(X)=0$ for every $X \in \bar{b}$. Then $\alpha$ becomes a root of $g^{\prime}$. Thus we can regard that $\Delta_{1}$ is a subset of the root system $\Delta$ of $g^{i}$ with respect to the Cartan subalgebra $\mathfrak{l}^{\prime}$. Then, $\bar{\Delta}_{1}$ is the root system of $\overline{\mathfrak{a}}$ with respect to $\bar{b}$. Let

$$
\Pi_{1}=\left\{\alpha_{1}, \ldots, \alpha_{k}\right\}, \bar{\Pi}_{1}=\left\{\bar{\alpha}_{1}, \ldots, \bar{\alpha}_{k}\right\}
$$

be fundamental root systems of $a$, it respectively. Then

12) This is seen analogously as in the formula (2). 


$$
\Pi=\left\{\alpha_{1}, \ldots, \alpha_{k}, \bar{\alpha}_{1}, \ldots, \bar{\alpha}_{k}\right\} .
$$

is a fundamental root system of $\mathfrak{g}^{\prime}$.

Now let $\left\{A_{1}, \ldots, d_{k}\right\}$ be the fundamental weight system of a determined by $\left\{\alpha_{1}, \ldots, \alpha_{k}\right\}$. Then $\left\{\overline{1}_{1}, \ldots, \bar{A}_{k}\right\}$ is the fundamental weight system of $\bar{a}$ determined by $\left\{\bar{\alpha}_{1}, \ldots, \bar{\alpha}_{k}\right\}$. Then $\left\{A_{1}, \ldots, A_{k}, \bar{A}_{1}, \ldots, \bar{A}_{k}\right\}$ the fundamental weight system of $g^{c}$ determined by $\left\{\alpha_{1}, \ldots, \alpha_{k}, \bar{\alpha}_{1}, \ldots, \bar{\alpha}_{k}\right\}$. Now let $\rho_{1}$, $\ldots, \mu_{k}$ be the fundamental irreducible representations of $a$ with highest weights $A_{1}, \ldots, A_{k}$ respectively. Then $\bar{\rho}_{1}, \ldots, \bar{\rho}_{k}$ are the fundamental irreducible representations of $i$ with highest weight $\bar{A}_{1}, \ldots, \bar{A}_{k}$ respectively. Let us regard $\rho_{1}, \ldots, \rho_{k}, \bar{\rho}_{1}, \ldots, \bar{\rho}_{k}$ as representation of $q^{c}$. Then $\mu_{1}, \ldots, \mu_{k}$, $\bar{\rho}_{1}, \ldots, \bar{\rho}_{k}$ are the fundamental irreducible representation of $\mathrm{g}^{\prime}$ determined by $\alpha_{1}, \ldots, \alpha_{k}, \bar{\alpha}_{1}, \ldots, \bar{\alpha}_{k}$.

Now let $(\rho, V)$ be an irreducible representation of $₫$ with highest weight 1 . Put $.1=\sum_{i=1}^{k} m_{i} 1_{i}+\sum_{i=1}^{k} m_{i}^{\prime} \bar{\pi}_{i} . \quad$ Then, by Theorem 2 we see that $\rho$ is self-conjugate if ane only if $m_{i}=m_{i}^{\prime}(i=1, \ldots, k)$. Moreover, if $\rho$ is self-conjugate, then the index of $\rho$ is necessarily equal to 1 . Now let us extend the representation $(\rho, V)$ to the representation of $\mathfrak{g}^{\prime}$ (this representation is also denoted by $(\rho, V)$ ). Let $\sigma_{1}, \sigma_{2}$ be the induced irreducible representation of $a, \pi$ respectively by $\rho$. Then the highest weight of $\sigma_{1}, \sigma_{2}$ are $\Sigma m_{i} 1_{i}, \Sigma m_{i}^{\prime} T_{i}$ respectively. In fact, let $x \in V_{\Lambda}, x \neq 0$. Then we have ${ }(H) x=A(H) x,\left(H \in \mathfrak{h}^{\prime}\right)$. If we put $H=B_{1}+\bar{B}_{2}$, $\left(B_{1}, B_{2} \in \mathfrak{b}\right)$, then

$$
\rho(H) x=\left(\Sigma m_{i} A_{i}\left(B_{1}\right)+\Sigma m_{i}^{\prime} \overline{1}_{i}\left(\bar{B}_{2}\right)\right) x .
$$

If $H \in \mathfrak{b}$, then $B_{2}=0$, and we have

$$
\rho(H) x=\left(\Sigma m_{i} \Lambda_{i}(H)\right) x
$$

Thus $\Sigma m_{i} A_{i}, \Sigma m_{i}^{\prime} \bar{A}_{i}$ are weights of $\sigma_{1}, \sigma_{2}$. If $\sigma_{1}$ has a weight.$^{\prime}$ higher than $\Sigma m_{i} 1_{i}$, then $1^{\prime}+\Sigma m_{i}^{\prime} \bar{A}_{i}$ is a weight of $\rho$ higher than .1 . This is a contradiction. Hence $\Sigma m_{i} l_{i}, \Sigma m_{i}^{\prime} \bar{\Lambda}_{i}$ are highest weights.

Now $\mathfrak{a}_{R} \cong \bar{a}_{R}$ by the canonical isomorphism $X \rightarrow \bar{Y}\left(X \in a_{R}\right)$. If we identify $\mathfrak{a}_{R}$ and $\bar{a}_{R}$ under this isomorphism, then $\sigma_{1}, \sigma_{2}$ can be regarded as the representations of $a_{R}$. Then we have $m_{i}=m_{i}^{\prime}(i=1, \ldots, k)$ if and only if $\bar{\sigma}_{1} \sim \sigma_{: 2}$ as the representation of $a_{k}$. Thus we have the following 
THEOREM 3. Let $g$ be a simple Lie algebra over $R$ such that $\mathrm{g}^{c}$ is not simple. Let $\mathrm{g}^{\mathrm{f}}=\mathfrak{a}+\overline{\mathrm{a}}$ be the decomposition of $\mathrm{g}^{\mathrm{f}}$ into simple ideals. Let $\rho$ be an irreducible representation of $\mathrm{g}$, and $\sigma_{1}, \sigma_{2}$ be the induced irreducible representation of $\mathfrak{a}, \bar{a}$ by the extension of $\rho$ to $g^{i}$. If we identify $a_{R}, \bar{a}_{R}$ under the isomorphism $X \rightarrow \bar{X}(X \in \mathfrak{a})$, we can regard $\sigma_{1}, \sigma_{2}$ as representations of $\mathfrak{a}_{R}$. Then $\rho \sim \bar{\rho}$ if and only if $\bar{\sigma}_{1} \sim \sigma_{2}$ as representations of $a_{R}$. If $\rho \sim \bar{\rho}$, then the index of $\rho$ is 1 .

\section{$\S 12$. An application to self-contragradient representations}

Let $\tilde{\mathrm{g}}$ be a semi-simple Lie algebra over $C$. Let $(\rho, V)$ be a representation of $\widetilde{a}$. Let us denote by $\left(\rho^{*}, V^{*}\right)$ the contragradient representation of $(\mu, V)$, i.e. $V^{*}$ is the dual vector space of $V$ and $\rho^{*}$ is given by $\rho^{*}(X)=-{ }^{t} \rho(X)$ for any $X \in \tilde{\mathrm{g}} . \quad(\rho, V)$ is called self-contragradient if $\rho \sim \rho^{*}$.

Now let $g$ be a compact real form of $\bar{\alpha}$. Let us denote by $\rho \mid g$ the restriction of a representation $\rho$ to $g$. Then, since any continuous representation of a compact group is equivalent to a representation by unitary matrices, we have $(\rho \mid g)^{*} \sim \overline{(\rho \mid g)}$ for any representation $\rho$ of $\AA$. Moreover, two representations of $\tilde{g}$ are equivalent if and only if their restrictions to $g$ are equivalent. Since we have $\rho^{*} \mid g \sim(\rho \mid g)^{*}$, the problem of the self-contragradience of a representation $\rho$ of $\tilde{\mathrm{g}}$ is reduced to that of the self-conjugateness of $\rho \mid g$, i.e. we have $\rho \sim \rho^{*}$ if and only if $(\rho \mid g) \sim \rho \mid g$. Then we can apply theorem 2 . Let $\mathfrak{h}$ be a Cartan subalgebra of $g$. Let $\alpha_{1}, \ldots, \alpha_{l}$ be any fundamental root system of $\tilde{\mathrm{g}}$ with respect to $\mathfrak{h}^{\prime}$, and $A_{1}, \ldots, A_{l}$ be the fundamental weight system determined by $\alpha_{1}, \ldots, \alpha_{l}$, and $\rho_{1}, \ldots, \rho_{l}$ be the irreducible representations of $\tilde{a}$ whose highest weights are $A_{1}, \ldots, A_{l}$ respectively.

Then, by theorem 2 , there exists a involutive permutation $\sigma$ of $1, \ldots, l$ such that $\rho_{\sigma(i)}^{*} \sim \rho_{i}(i=1, \ldots, l)$.

Now, let $\rho$ be an irreducible representation of $\tilde{\mathfrak{g}}$ with the highest weight $A=\sum_{i=1}^{l} m_{i} A_{i}$. Then the highest weight of $\rho^{*}$ is given by $\sum_{i=1}^{l} m_{\sigma(i)} A_{i}$. Hence $\rho$ is self-contragradient if and only if $m_{i}=m_{\Im(i)}(i=1, \ldots, l)$.

\section{BIBLIOGRAPHY}

[1] É. Cartan, Les groupes projectifs continus réels qui ne laissent invariante aucune 
multiplicité plane, Journ. Math. pures et appl., t. 10 (1914), p. 149-186, or OEuvres complètes t. I, vol. 1, p. 493--530.

[2] Séminaire "Sophus Lie”, École Normale Supérieure (1954-195̄̄).

\section{The College of General Education}

University of Tokyo 